\title{
NOTA CIENTIFICA
}

\section{Notas sobre la reproducción en cautiverio de Crocodylus acutus} (Cuvier, 1807) en el Perú

\section{Observations about reproduction on captivity of Crocodylus acutus (Cuvier, 1807) in Peru}

\author{
Oswaldo Pérez ${ }^{1}$ y Armando H. Escobedo-Galván ${ }^{2,3}$
}

Presentado: $03 / 11 / 2005$

Aceptado: 23/01/2006

\section{Resumen}

Durante los años 2001 y 2002 se realizaron observaciones sobre la reproducción de Crocodylus acutus (Cuvier, 1807) en el Centro de Acuicultura La Tuna Carranza, localizado en Puerto Pizarro, departamento de Tumbes. El tamaño mínimo de una hembra anidando fue de 2,30 m. El porcentaje de viabilidad y natalidad fue de $61,71 \%$ y $93,15 \%$ respectivamente.

Palabras claves: Crocodylus acutus, Cocodrilo de Tumbes, ecología reproductiva, Perú.

\section{Abstract}

Observations about reproduction of Crocodylus acutus (Cuvier, 1807) were recorded during the years 2001 and 2002 in the Fishery Center «La Tuna Carranza» (Puerto Pizarro, Tumbes, Peru). The minimum size of a female nesting was $2,30 \mathrm{~m}$. The percentage of viability and birthrate/natality were $61,71 \%$ and $93,15 \%$, respectively.

Keywords: Crocodylus acutus, Tumbes Crocodile, reproductive ecology, Peru.

Debido a la importancia comercial y al deterioro de sus poblaciones naturales las especies del orden Crocodylia, desde la década de 1960, han sido estudiadas en sus características reproductivas, con la finalidad de mejorar las técnicas de manejo en cautiverio. Allsteadt (1994) mencionó que la reproducción es uno de los factores vitales que afectan a las poblaciones silvestres de cocodrilos, por lo que conocer estos factores puede ayudar a su conservación. Sin embargo, el estudio de las poblaciones naturales in situ es difícil, debido entre otras cosas a la drástica disminución de

${ }^{1}$ Centro de Acuicultura La Tuna Carranza, Fondo Nacional de Desarrollo Pesquero. Dirección: GRAU 722-Tumbes.

E-mail Oswaldo Pérez: oswper@terra.com.pe

${ }^{2}$ Escuela de Ciencias Biológicas, Universidad Nacional, Heredia, Costa Rica.

${ }^{3}$ Dirección actual: Julio Cervantes 561, Col. San Lorenzo Oriente, C.P. 25060, Saltillo, Coahuila, México. E-mail Armando Escobedo: elchorvis@gmail.com ellas, por este motivo gran parte de la información generada sobre la reproducción de Crocodylus intermedius, C. moreletii y Caiman latirostris, ha sido obtenida en cautiverio (Huerta, 1986; Thorbjarnarson y Hernández, 1993; Verdade, 1995; CasasAndreu y Barrios-Quirós, 1997).

Crocodylus acutus, conocido como Cocodrilo Americano o Cocodrilo de Tumbes, se encuentra en peligro de extinción (CITES, 2003). Esto ha generado el establecimiento de zoocriaderos con fines de reintroducción, repoblación, ecoturismo y educación ambiental (Sánchez, 1999; Pérez, 2002). En Perú, a partir de 1996 el Fondo Nacional de Desarrollo Pesquero (FONDEPES) estableció en el Centro de Acuicultura La Tuna Carranza, localizado en Puerto Pizarro, departamento de Tumbes, un criadero experimental del Cocodrilo de Tumbes con la finalidad de revertir la situación de la especie en la cuenta del río Tumbes y cuencas aledañas; obteniendo sus 
Tabla 1. Características físicas de los nidos colocados en los años 2001 y 2002, en el criadero experimental del Centro de Acuicultura La Tuna Carranza (Puerto Pizarro, Tumbes, Perú)

\begin{tabular}{|c|c|c|c|c|c|c|c|}
\hline \multirow[b]{2}{*}{ V ariable } & \multicolumn{3}{|c|}{ Época 2001} & \multicolumn{4}{|c|}{ Época 2002} \\
\hline & 1 & 2 & 3 & 1 & 2 & 3 & 4 \\
\hline Forma del nido & olla & olla & olla & olla & olla & olla & olla \\
\hline Tipo de suelo & arenoso & arenoso & arenoso & arenoso & arenoso & arenoso & arenoso \\
\hline A ncho del nido $(\mathrm{cm})$ & 120 & 50 & 80 & 120 & 50 & 50 & 100 \\
\hline Largo del nido $(\mathrm{cm})$ & 60 & 40 & 70 & 60 & 40 & 50 & 90 \\
\hline Profundidad del nido $(\mathrm{cm})$ & 60 & 60 & 20 & - & - & - & - \\
\hline Número de huevos & 25 & 23 & 27 & - & - & - & - \\
\hline Distancia al agua (cm) & 130 & 50 & 70 & 1300 & 110 & 50 & 800 \\
\hline Distancia al arbusto más cercano $(\mathrm{cm})$ & ) 50 & 20 & 300 & 50 & 20 & 300 & 120 \\
\hline Temperatura del nido $\left({ }^{\circ} \mathrm{C}\right)$ & - & & - & 28,4 & 28,5 & 28 & 28 \\
\hline
\end{tabular}

primeros resultados a partir del año 2001 (Escobedo, 2004; Pérez y Escobedo, 2005). El objetivo de la presente nota es dar a conocer las observaciones sobre la ecología reproductiva del Cocodrilo de Tumbes en cautiverio en Perú.

\section{Tamaño y edad de las hembras reproductoras activas}

Durante los meses de agosto a octubre se observó la postura de huevos, mientras que en diciembre y enero se observó la eclosión. El tamaño y peso promedio de hembras anidando para el año 2001 fue de 2,41 $\pm 10,15 \mathrm{~m}$ y $52,67 \pm 5,69 \mathrm{~kg}$, respectivamente y de 2,49 \pm 7,39 m y 59,5 $\pm 5,07$ para el año 2002 . El tamaño mínimo fue de 2,30 m. El tamaño promedio observado en Perú es menor al observado Thorbjarnarson y Hernández (1993; X= $3,06 \mathrm{~m}$ ) para $C$. intermedius, y por Thorbjarnarson $(1996 ; X=2,66 \mathrm{~m})$ y CasaAndreu (2003; 2,90 \pm 0,24 m) para C. acutus. Por otro lado, se observó que dos de las hembras que anidaron en el 2001, también lo realizaron durante el 2002. Casas-Andreu y Barios-Quiroz (1997) observaron un intervalo de anidación de uno a cuatro años. Esto se debe a que las especies pueden cambiar su comportamiento reproductivo desfasándolo temporalmente como respuesta al medio.

\section{Características de los nidos}

Entre los años 2001 y 2002 se llevo acabo la construcción de siete nidos. El promedio de huevos por nido fue de $25 \pm 2$ huevos. Los nidos presentaron una profundidad promedio de 46,67 $\pm 23,09 \mathrm{~cm}$. Las características físicas del nido se observan en el tabla 1.

La viabilidad promedio de los huevos colocados en el 2001 fue de 61,71\% (Tabla 2), siendo menor al promedio observado por Casas-Andreu $(2003 ; 81 \pm 21 \%)$. La natalidad promedio en el 2001 fue de $93,15 \%$, siendo similar al porcentaje observado por CupulMagaña y Aranda-Mena (2005) en Puerto Vallarta, México. La baja viabilidad puede ser asociada a que hembras jóvenes tienden a presentar una baja fertilidad y al mismo tiempo darse un abandono del nido por parte de las hembras afectando la sobrevivencia de las crías (Magnusson, 1980; Mazzotti, 1989).

Tabla 2. Porcentaje de viabilidad y natalidad de los nidos colocados durante el año 2001.

\begin{tabular}{lcccccc}
\hline Nido & $\begin{array}{c}\text { Longitud hembra } \\
\text { LT (cm) }\end{array}$ & $\begin{array}{c}\text { Número de } \\
\text { huevos }\end{array}$ & $\begin{array}{c}\text { Huevos } \\
\text { Infértiles }\end{array}$ & $\begin{array}{c}\text { Crías } \\
\text { M uertas }\end{array}$ & $\begin{array}{c}\text { Viabilidad } \\
\text { (\%) }\end{array}$ & $\begin{array}{c}\text { N atalidad } \\
\text { (\%) }\end{array}$ \\
\hline 1 & 250 & 25 & 4 & 1 & 84 & 95,24 \\
2 & 230 & 27 & 22 & 0 & 18,52 & 100 \\
3 & 243 & 23 & 4 & 3 & 82,61 & 84,21 \\
\hline
\end{tabular}


En las próximas épocas de anidación podría esperarse un aumento en el número de hembras reproductoras activas, así como en el número de huevos, el porcentaje de viabilidad y natalidad, debido ha que se ha observado una relación positiva entre estas variables con la longitud de las hembras (Thorbjarnarson, 1996; Casas-Andreu, 2003).

\section{Agradecimientos}

A Zandro Carrión por todo su apoyo y colaboración en la crianza del cocodrilo de Tumbes.

\section{Literatura citada}

Allsteadt, J. 1994. Nesting ecology of Caiman crocodilus in Caño Negro, Costa Rica. Journal of Herpetology 28(1): 12-19.

Casas-Andreu, G. 2003. Ecología de la anidación de Crocodylus acutus (Reptilia: Crocodylidae) en la desembocadura del río Cuitzmala, Jalisco, México. Acta Zoológica Mexicana (n.s.) 89: 111-128.

Casas-Andreu, G. y G. Barrios-Quirós. 1997. Nuevos aportes a la anidación (1990-1993) de Crocodylus moreletii en cautiverio en Tabasco, México. pp. 21-25. Memorias de las $4^{\text {ta }} \mathrm{Re}-$ unión Regional del Grupo de Especialistas de Cocodrilos de América Latina y el Caribe. Centro Regional de Innovación Agroindustrial, S.C. Villahermosa, Tabasco.

Convenio Internacional para el Trafico de Especies Amenazadas de Flora y Fauna (CITES). 2003. [en línea]. <http://www.cites.org/eng/append/ appendices.shtml > [10 Agosto 2004].

Cupul-Magaña, F. G. y O. S. Aranda-Mena. 2005. Éxito de eclosión del cocodrilo americano (Crocodylus acutus) y la tortuga golfina (Lepidochelys olivacea) en Puerto Vallarta, Jalisco, México. Revista Electrónica de Veterinaria REDVET VI(10): 1-7.
Escobedo, A. H. 2004. Avances en el conocimiento y el estado actual de conservación del Cocodrilo de Tumbes (Crocodylus acutus Cuvier, 1807). Revista peruana de biología 11(2): 203-208.

Huerta, P. 1986. Etología, reproducción y biometría del cocodrilo (Crocodylus moreletii Dumeril, Bribon y Dumeril), en cautiverio. Tesis de Licenciatura. Instituto Politécnico Nacional, México. 69 p.

Magnusson, W. E. 1980. Hatching and creche formation by Crocodylus porosus. Copeia 1980(2): 359362.

Mazzotti, F. J. 1989. Factors affecting the nesting success of American crocodile, Crocodylus acutus, in Florida Bay. Bulletin of Marine Science 44(1): 220-228.

Pérez, O. 2002. Centro de acuicultura la Tuna Carranza, Avances 2002. Informe Técnico. FONDEPES. $10 \mathrm{p}$.

Pérez, O., Z. Carrión y A. Luján. 2005. Avances de la crianza en cautiverio de Crocodylus acutus «Cocodrilo Americano». Informe Técnico. FONDEPES. 34 p.

Pérez, O. y A. H. Escobedo. 2005. Observaciones biométricas de Crocodylus acutus (Cuvier, 1807) recién nacidos en cautiverio, Tumbes, Perú. Revista peruana de biología 12(1): 171172.

Sánchez, J. J. 1999. Aspectos básicos del manejo de poblaciones cautivas de Caiman crocodilus fuscus y Crocodylus acutus, en Costa Rica. Tesis de Licenciatura. Universidad Nacional, Heredia, Costa Rica. 174 p.

Thorbjarnarson, J. B. y G. Hernández. 1993. Reproductive ecology of the Orinoco Crocodile (Crocodylus intermedius) in Venezuela. I. Nesting ecology and egg and clutch relationships. Journal of Herpetology 27(4): 363-370.

Verdade, L. M. 1995. Biología reproductiva do Jacaréde-Papo-Amarelo (Caiman latirostris) em Sao Paulo, Brasil. pp. 57-79. In Larriera, A. \& L. M. Verdade (Eds.). La Conservación y el manejo de caimanes y cocodrilos de América Latina. Vol. 1. Fundación Banco Bica, Santo Tomé, Santa Fe, Argentina. 
| 